\title{
Sustainable groundwater management with tradable permits
}

\author{
J-C. Pereau ${ }^{a}$, L. Mouysset ${ }^{b}$ and L. Doyen ${ }^{b}$ \\ ${ }^{a}$ GREThA UMR 5113, Université de Bordeaux, Avenue Léon Duguit, 33608 Pessac CEDEX, France \\ ${ }^{\mathrm{b}}$ CNRS, UMR 5113 GREThA, Université de Bordeaux, avenue Léon Duguit 33608 Pessac cedex, France \\ Email: lauriane.mouysset@u-bordeaux.fr
}

\begin{abstract}
This article studies the sustainability of market-based instrument such as tradable permits for the management of a renewable aquifer used for agriculture production. In a dynamic hydro-economic model, a manager aims to satisfy different constraints in terms of food security and individual profit within a tradable permit scheme. We identify analytically the viability kernel that defines the states of the resource yielding inter-temporal feasible paths able to satisfy the set of constraints over time.
\end{abstract}

Keywords: Groundwater, agriculture, irrigation, food security, individual permits 


\section{INTRODUCTION}

Groundwater resources are under extreme pressure worldwide. About $20 \%$ of the world's aquifers are overexploited (WWAP, 2015). Increasing demand for agricultural products to satisfy the needs of a growing population is the main driver behind water use together with drinking water(FAO, 2014). Irrigation is the principal user of aquifers and inefficient use of water for crop production depletes aquifers. As groundwater is a common property resource, aquifer depletion can occur when water extraction from myopic agents always exceeds its natural recharge. Regulation is needed to ensure a viable management of renewable aquifers and to sustain the exploitation of water resource.

The use of market-based instruments, such as transferable permits, has been proposed as a promising way to manage efficiently a groundwater aquifer for irrigated agriculture (Latinopoulos and Sartzetakis, 2015). Transferability ensures that water goes to the use with the higher value within the agriculture sector. The market price for permits is used as signal to allocate water between farmers. Efficiency means that the amount of water extraction that a regulator allocates between farmers provides the maximum amount of food production. As pointed out by Roumasset and Wada (2012), there is a closed connection between groundwater and biological renewable resources, like fishes. The natural recharge rate is analogous to the biological growth rate for fish, except that the growth rate depends on the current stock while the natural recharge is exogenous. Water permits in aquifers are also analogous to individual and transferable quotas in fisheries (Pereau et al, 2012).

There has been considerable research in the development of hydro-economic model in the follow-up of the dynamic model of Gisser and Sanchez (1980). The state of art of this literature including management issues and game theoretical models has been addressed in Rubio and Casino (2001), Madani and Dinar (2013) and Tomini (2014). In the design of a feasible water market, Ballestero et al (2002) suggest that water reform will be more equitable and therefore politically acceptable if the allocation of permits is based on historical rights. It appears also important to ensure a minimum amount of profit to each farmer. But the main challenge remains to secure food supply for a large part of the population in many countries. This suggests a multi criteria approach to manage aquifers sustainably and to balance economic efficiency and equity. This suggests to define precautionary level of the resource along time in order to fulfilled some constraints and objectives.

This paper describes the management of an aquifer by a water agency using a transferable permit scheme facing myopic heterogenous farmers in the dynamic Gisser and Sanchez (1980) model. The analysis of our hydroeconomic model relies on the weak invariance (Aubin, 1990) or viable control method (Clarke et al, 1995). This approach focuses on identifying inter-temporal feasible paths within a set of desirable objectives or constraints (Béné et al, 2001). This framework has been applied to renewable resources management and especially to the regulation of fisheries (Martinet et al, 2007; Doyen and Pereau, 2012).

Section 2 is devoted to the description of the dynamic hydro-economic model and the objectives of the water agency. Section 3 characterizes the feasible resource states and water policies under several constraints.

\section{THE HYDRO-ECONOMIC MODEL}

\subsection{The resource dynamics}

An aquifer is described by its state variable (ie the height of water) $H(t) \in\left[0 ; S_{L}\right]$ at time $t$ where $S_{L}$ stands for the height of the ground surface. At $H(t)=0$, the aquifer is empty, at $H(t)=S_{L}$ the aquifer is full. The height of water increases with constant rainfalls $R>0$ and decreases because of extraction $W(t)$ dedicated to agriculture by $n$ farmers with $W(t)=\sum_{i=1}^{n} w_{i}(t)$. We suppose that a proportion $\mu$ of the water used for irrigation comes back to the aquifer. Then total extraction is $(1-\mu) W$ where $0<\mu<1$ stands for the non-absorption coefficient. $A$ stands for the area of the aquifer and $S$ the storage coefficient.

Based on Gisser-Sanchez (1980), the dynamics of the resource is

$$
\begin{aligned}
H(t+1) & =H(t)+\frac{1-\mu}{A S}\left(W_{R}-W(t)\right) \\
H(0) & =H_{0}
\end{aligned}
$$

where $W_{R}=\frac{R}{1-\mu}$ stands for the level of extraction which maintains a constant table water $(H(t+1)=H(t))$. If the extraction is too high $\left(W(t)>W_{R}\right)$, the rainfalls are lower than the extraction compensate the extraction and the aquifer volume decreases. 
J-C. Pereau, L. Mouysset, L. Doyen Sustainable groundwater management with tradable permits

\subsection{The water permit market}

A set of $n$ heterogeneous farmers $i$ use water denoted by $w_{i}$ from the aquifer to irrigate their crops. The individual profit of farmer $i$ is given by

$$
\pi_{i}(t)=p_{y} y_{i}(t)-c(t) w_{i}(t)-m(t)\left(w_{i}(t)-w_{i}^{-}(t)\right)
$$

The first term of eqn. (2) refers to the total income with $p_{y}$ the price of the agricultural product and $y_{i}(t)$ the individual production which is assumed to be a quadratic form of the water use $w_{i}(t)$

$$
y_{i}(t)=a_{i} w_{i}(t)-\frac{b_{i}}{2} w_{i}^{2}(t)
$$

where $a_{i}, b_{i}>0$ are technical parameters of production. Marginal productivity is positive and decreasing. Individual production reaches a maximum for $\overline{w_{i}}=a_{i} / b_{i}$ yielding $\overline{y_{i}}=a_{i}^{2} / 2 b_{i}=a_{i} \overline{w_{i}} / 2$. It implies that individual water extraction $w_{i} \in\left[0, \overline{w_{i}}\right]$. The maximum amount of water consumption is $\bar{W}=\sum_{i=1}^{n} \overline{w_{i}}$ and the maximum amount of production is then $\bar{Y}=\sum_{i=1}^{n} \overline{y_{i}}$. Farmers are supposed to be price takers on the product market.

The second term of eqn. (2) refers to the extraction cost. The unitary cost $c(t)$ is given by

$$
c(t)=c_{1}\left(S_{L}-H(t)\right)=c_{0}-c_{1} H(t)
$$

where $c_{0}=c_{1} S_{L}$ stands for a fixed cost and $c_{1}$ is the marginal pumping cost. The unitary cost increases when the level of the water table decreases. The third term of eqn. (2) refers to the transferable permit market where $m(t)$ stands for the unitary price of the water permit. It is assumed water extraction is managed by a regulator who allocates transferable water permits to the $n$ farmers at the beginning of each period $t$. After receiving their water entitlements $w_{i}^{-}(t)$ free of charge at each period, farmers decide whether to buy or sell water permits to other farmers and how many to trade, based on their annual water uses $w_{i}(t)$. It is assumed that water permits are not transferable through time, that is, no banking or borrowing of water permits is allowed.

The total water supply (ie the global amount of water allocated by the regulator) is computed as follow

$$
W(t)=\sum_{i=1}^{n} w_{i}^{-}(t)
$$

We assume that the individual allocation rule $w_{i}^{-}(t)=\kappa_{i} W(t)$ is egalitarian and ensures that every farmer receives at each period with $\kappa_{i}=\frac{1}{n}$. The aggregated water quota demand depends on the optimal individual quotas, which emerges from the maximisation of individual profits

$$
\max _{w_{i}} \pi_{i}(t)
$$

First order conditions give the optimal individual water demand

$$
w_{i}^{*}(t)=\left(\frac{a_{i}}{b_{i}}-\frac{c_{0}}{p_{y} b_{i}}-\frac{m(t)}{p_{y} b_{i}}\right)+\frac{c_{1}}{p_{y} b_{i}} H(t)
$$

We deduce the aggregate water demand

$$
W^{*}(t)=\sum_{i=1}^{n} w_{i}^{*}(t)=\bar{W}-\beta \frac{c_{0}}{p_{y}}-\beta \frac{m(t)}{p_{y}}+\beta \frac{c_{1}}{p_{y}} H(t)
$$

with

$$
\bar{W}=\sum_{i=1}^{n} \frac{a_{i}}{b_{i}} ; \beta=\sum_{i=1}^{n} \frac{1}{b_{i}}
$$

The clearing market condition on the water market implies the equilibrium water price $m^{*}(t)$

$$
m^{*}(t)=\frac{p_{y}}{\beta}(\bar{W}-W(t))-c_{0}+c_{1} H(t)
$$

This expression confirms the economic intuitions with $\frac{\partial m^{*}}{\partial W}<0$ and $\frac{\partial m^{*}}{\partial H}>0$. An increase in the water supply faced to an unchanged demand implies a decrease in the water price. When the height of the water table is high, water extraction increases and thus the demand of permits, pushing up the water price. 
J-C. Pereau, L. Mouysset, L. Doyen Sustainable groundwater management with tradable permits

\section{THE REGULATOR CONSTRAINTS}

\subsection{The tradable water permit constraint}

If a positive permit demand exist, then the price of the water permit $m^{*}(t)$ is positive $0 \leq m^{*}(t)$. It yields a state-control constraint

$$
W(t) \leq W_{M}(H(t))=\left(\bar{W}-\frac{\beta c_{0}}{p_{y}}\right)+\frac{c_{1} \beta}{p_{y}} H(t)
$$

The tradable water permit constraint entails a higher limit on the value of the total water extraction $W(t)$. This superior bound is an increasing function of the state variable $H(t)$ ). This bound depends on the economic parameters of farmers.

\subsection{The food security constraint}

To deal with the questions related to food security, the aggregate production of the agricultural sector has to satisfy a minimum threshold $Y_{l i m} \leq Y^{*}(t)$

By substitution, we obtain

$$
w_{i}^{*}(t)=\frac{1}{b_{i}}\left(a_{i}-\left(\frac{\bar{W}-W(t)}{\beta}\right)\right)
$$

that yields the optimal individual production and then the aggregated production

$$
Y^{*}(t)=\bar{Y}-\frac{1}{2 \beta}(\bar{W}-W(t))^{2}
$$

with $\bar{Y}=\sum_{i=1}^{n} \overline{y_{i}}$.

The aggregated production constraint $Y_{\text {lim }} \leq Y^{*}(t)$ implies that the water supply $W(t)$ is bounded by an inferior limit $W_{F S}$

$$
W_{F S}=\bar{W}-\sqrt{2 \beta\left(\bar{Y}-Y_{\lim }\right)} \leq W(t)
$$

where $W_{F S}$ is constant and independent from the state variable. Note that the food security is positive $W_{F S} \geq$ 0 for a minimum amount of production $Y_{\lim } \geq Y_{\lim }^{\min }$ with $Y_{\lim }^{\min }=\bar{Y}-\left(\bar{W}^{2} / 2 \beta\right)$.

\subsection{The individual profit constraint}

To maintain their activity, farmers need to make profit. This can be translatable within an individual profit constraint $\pi_{\lim } \leq \pi_{i}^{*}(t)$ which can be rewritten as

$$
\pi_{i}^{*}(t)=\frac{p_{y}}{2 b_{i}}\left(a_{i}-\left(\frac{\bar{W}-W}{\beta}\right)\right)^{2}+w_{i}^{-} m^{*}
$$

or after substitution

$$
A W(t)+B+\frac{C}{W(t)} \leq H(t)
$$

with

$$
A=\frac{p_{y}}{\beta c_{1}}\left(1-\frac{1}{2 b_{i} \kappa_{i} \beta}\right) ; B=\frac{c_{0}}{c_{1}}-\frac{p_{y}}{\beta c_{1}}\left(\left(1-\frac{1}{\beta \kappa_{i} b_{i}}\right) \bar{W}+\frac{a_{i}}{\kappa_{i} b_{i}}\right) ; C=\frac{\pi_{l i m}}{c_{1} \kappa_{i}}-\frac{p_{y}}{c_{1} \kappa_{i} 2 b_{i}}\left(a_{i}-\frac{\bar{W}}{\beta}\right)^{2}
$$

Eq 16 implies a quadratic equation in $W$ such that $A W^{2}+(B-H) W+C \leq 0$. It turns out that for plausible values of the parameters ( $\beta$ gets a high value) $A>0$ while $C$ is positive for a minimum value of the profit $\pi_{l i m} \geq \pi_{l i m}^{m i n}$ with $\pi_{l i m}^{\min }=\frac{p_{y}}{2 b_{i}}\left(a_{i}-\frac{\bar{W}}{\beta}\right)^{2}$ Hence, the individual profit constraint $\pi_{\lim } \leq \pi_{i}^{*}(t)$ yields

$$
W_{I P}^{\text {inf }}(H(t)) \leq W(t) \leq W_{I P}^{\text {sup }}(H(t))
$$


J-C. Pereau, L. Mouysset, L. Doyen Sustainable groundwater management with tradable permits

with, on $\left.W=] 0 ; \sqrt{\frac{C}{A}}\right]$ solution $W_{I P}^{i n f}(H)$ and on $W=\left[\sqrt{\frac{C}{A}} ;+\infty\left[\right.\right.$ solution $W_{I P}^{\text {sup }}(H)$ with

$$
W_{I P}^{\text {inf }}(H)=\frac{-B+H-\sqrt{(B-H)^{2}-4 A C}}{2 A} ; W_{I P}^{\text {sup }}(H)=\frac{-B+H+\sqrt{(B-H)^{2}-4 A C}}{2 A}
$$

under the condition $(B-H)^{2}-4 A C>0$

This individual profit constraint bounds the extraction $W$ with the inverse of a hyperbolic function of $H(t)$.

\section{THEORETICAL RESULTS}

\subsection{The resource threshold}

The dynamics of the aquifer given by eqn. (1) evolves within the combination of the water permit price constraint (11), the food security constraint and the individual profit constraint. Combining these three constraints gives

$$
\max \left(W_{F S}, W_{I P}^{\text {inf }}\right) \leq W \leq \min \left(W_{M}, W_{I P}^{\text {sup }}\right)
$$

This yields a constraint on the level of the water table

$$
H_{\lim } \leq H(t)
$$

where $H_{\text {lim }}$ emerges from the intersection of the different constraints. More precisely, we define $H_{\text {lim }}$ the threshold on $H$ emerging from the most restrictive two-by-two intersections of the three constraints.

\subsection{The dynamic context}

The resource threshold $H_{\text {lim }}$ has to be verified within a dynamic context $H_{\text {lim }} \leq H(t+1)$. Using eq (1), we obtain:

$$
W(t) \leq W_{D}(H(t))=W_{R}+\frac{A S}{(1-\mu)}\left(H(t)-H_{\lim }\right)
$$

This dynamic constraint leads to a superior bound for the total water extraction $W(t)$. This superior limit is an affine and increasing function of the state variable $H(t)$.

This constraint is more stringent than the water permit constraint under the condition on the marginal pumping cost:

$$
c_{1}>\left(\frac{A S}{1-\mu}\right)\left(\frac{p_{y}}{\beta}\right)
$$

\subsection{The maximum production and profit objectives}

The function $W_{D}(H(t))$ is characterized by $\left(H_{\text {lim }}, W_{R}\right)$. This yields a limit case define by $H(t)=H_{\text {lim }}$ where the dynamic constraint becomes $W(t) \leq W_{R}$.

Satisfying simultaneously the food security constraint (ie $W_{F S} \leq W(t)$ ) and the constraint $W(t) \leq W_{R}$ implies

$$
W_{F S} \leq W_{R}
$$

This condition means that if the food security is too demanding, the water extraction requiring for the demanding production is higher than the rainfalls. The water table decreases towards 0 , and it is not possible to define any sustainable water extraction.

The maximum $Y_{\text {lim }}$ able to sustain a non-empty groundwater (denoted by $Y_{\text {lim }}^{\max }$ ) is defined for $W_{F S}=W_{R}$, yielding

$$
Y_{\text {lim }}^{\max }=\bar{Y}-\frac{\left(\bar{W}-W_{R}\right)^{2}}{2 \beta}
$$


J-C. Pereau, L. Mouysset, L. Doyen Sustainable groundwater management with tradable permits

For $H(t)=H_{\text {lim }}$, the production reaches its maximum $Y_{\text {lim }}^{\max }$ and the constraint (ie $W_{R}$ ) and the food security constraint (ie $W_{F S}$ ) are overlapped. Satisfying simultaneously the individual profit constraint (ie $W_{I P}^{\text {inf }}(H(t)) \leq W(t) \leq W_{I P}^{\text {sup }}(H(t))$ ) and the constraint (ie $W(t) \leq W_{R}$ ) implies $W_{I P}^{\text {inf }} \leq W_{R} \leq W_{I P}^{\text {sup }}$. Similarly to the food security constraint, this condition implies a limit for the individual profit constraint, ie a maximum $\pi_{\text {lim }}^{\max }$. Over this value, the profit constraint implies a production too demanding in water; it is not possible to define any viable extraction of groundwater.

The value $\pi_{l i m}^{\max }$ is the maximum achievable optimal profit with $W_{I P}=W_{R}$ when $H=H_{\text {lim }}$

$$
\pi_{l i m}^{\max }=\max \left(\pi_{l i m}^{*}\left(W_{R}, H_{l i m}\right)\right)
$$

After substitution, $\pi_{\text {lim }}^{\max }$ is given by

$$
\pi_{l i m}^{\max }=\frac{p_{y}}{2 b_{i}}\left(a_{i}-\frac{\bar{W}-W_{R}}{\beta}\right)^{2}
$$

It shows that at $H(t)=H_{\text {lim }}$, there is an unique intersection of the dynamic constraint $W_{R}$, the water market constraint $W_{M}$ and the profit constraint $W_{I P}$.

Since the dynamic constraint $W_{R}$ and the food security constraint $W_{F S}$ are overlapped, there is an unique intersection of all the 4 constraints when $H=H_{l i m}$ and $W=W_{R}$. The production $Y$ reaches its maximum $Y_{\text {lim }}^{\max }$ as well as the individual profit $\pi_{\text {lim }}^{\max }$, the groundwater height is constant as well as the allowed extraction.

\subsection{The viability kernel}

The dynamics of the aquifer given by (1) is taken into account in combination with the three constraints already used above to determine $H_{\text {lim }}$ together the dynamic constraint (20): $W(t) \leq W_{D}(H(t))$

In an infinite horizon context, the viability kernel can be defined as the set of initial situation $H_{0}$ such as it exists water extraction $W(t)$ and resources $H(t)$, satisfying the previous constraints, for any time between $t=0,1, \ldots T$.

We obtain the following proposition:

Proposition 1. Assume that $W_{F S} \leq W_{R}, Y_{\text {lim }}^{\min } \leq Y_{\text {lim }} \leq Y_{\text {lim }}^{\max }$ and $\pi_{\text {lim }}^{\min } \leq \pi_{\text {lim }} \leq \pi_{\text {lim }}^{\max }$, we obtain

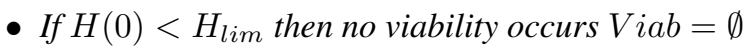

- If $H(0) \geq H_{\text {lim }}$ the viability kernel is Viab $=\left[H_{\mathrm{lim}}, S_{L}\right]$

\subsection{The viable quotas}

We are able to specify the viable quotas $W$ associated to the viable water tables.

Proposition 2. Considering that $W_{F S} \leq W_{R}$, the viable quotas associated to Viab $=\left[H_{\lim }, S_{L}\right]$ are

$$
W^{V i a b}=\left[\max \left(W_{I P}^{i n f}, W_{F S}\right), \min \left(W_{M}, W_{D}, W_{I P}^{\text {sup }}\right)\right]
$$

The figure 1 illustrates the state-control constraints and viable domain in a theoretical example where the four constraints defined in the section 4.4 are active.

\section{ACKNOWLEDGEMENT}

This study has been carried out with financial support from the French National Research Agency (ANR) as part of the project ADAPTEAU (ANR-11-CEPL-008) and in the frame of the Cluster of Excellence COTE (ANR-10-LABX-45).

\section{REFERENCES}

Aubin J-P (1990), A survey of viability theory, The SIAM Journal on Control and Optimization 28 (4) (1990) 749-788. 
J-C. Pereau, L. Mouysset, L. Doyen Sustainable groundwater management with tradable permits

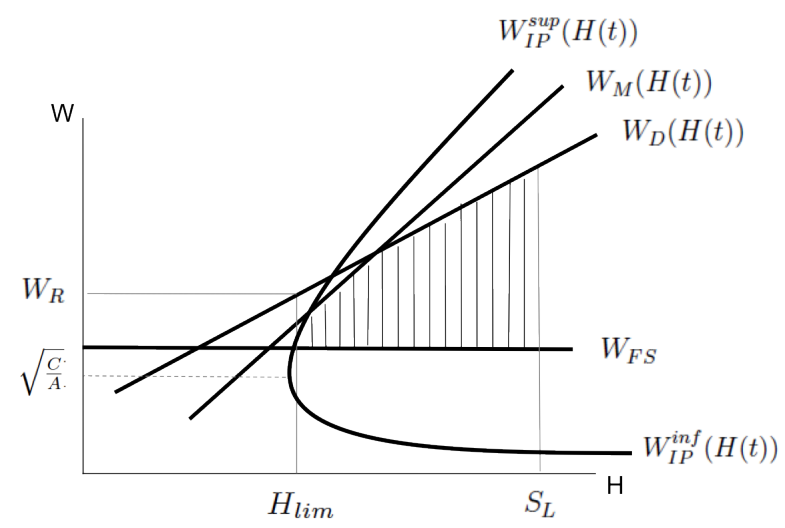

Figure 1. State-control constraints and viable domain. The x-axis stands for the height of the groundwater (the state variable), the $y$-axis stands for the extraction (the control variable). The shaded area corresponds to the viable domain, satisfying all the constraints as defined in the Prop 2.

Ballestero E., S. Alarcon and A. Garcia-Bernabeu (2002), Etablishing politically feasible water markets: a multi-criteria approach, Journal of Environmental Management, 65:411-429.

Clarke F.H., YU.S. Ledayev, R.J. Stern, P.R. Wolenski, (1995) Qualitative properties of trajectories of control systems: a survey, Journal of Dynamical and Control Systems, 1:1-48.

Doyen, L. and J-C. Pereau (2012) Sustainable coalitions in the commons, Mathematical Social Sciences 63(1):57-64.

FAO (2014) Building a common vision for sustainable food and agriculture: Principes and Approaches, Rome.

Gisser, M., and Sanchez, D.A., (1980) Competition versus optimal control in ground water pumping, Water Resource Research 31:638-642.

Latinopoulos D. and E.S. Sartzetakis (2015) Using Tradable Water Permits in Irrigated Agriculture, Environmental and Resource Economics, 60(3):349-370.

Madani K, and Dinar A. (2013) Exogenous regulatory institutions for sustainable common pool resource management: Application to groundwater, Water Resources and Economics, 2-3:57-76

Martinet V, O. Thébaud, L. Doyen, (2007) Defining viable recovery paths toward sustainable fisheries, Ecological Economics 64(2):411-422.

Roumasset J. and Wada C. (2012) The economics of grounwater, WP 2012-4 UHERO-University of Hawai

Rubio S.J. and Casino B. (2001) Competitive versus Efficient Extraction of a Common Property Resource: The Groundwater Case. Journal of Economic Dynamics and Control, 25(8), 1117-1137.

Tomini A. (2014) Is the Gisser and Sanchez model too simple top discuss the economic relevance of groundwater management? Water Resources and Economics, 6:18-29

WWAP (United Nations World Water Assessment Programme). 2015. Water for a Sustainable World. Paris, UNESCO. 